\title{
Associations of Ganglion Cell-Inner Plexiform Layer and Optic Nerve Head Parameters with Visual Field Sensitivity in Advanced Glaucoma
}

\author{
Haijian Hu Ping Li Xueqing Yu Wei Wei Hai He XuZhang \\ Jiangxi Clinical Research Center for Ophthalmic Disease, Jiangxi Research Institute of Ophthalmology and \\ Visual Science, Affiliated Eye Hospital of Nanchang University, Nanchang, China
}

\section{Keywords}

Ganglion cell-inner plexiform layer - Optic nerve head .

Visual field · Associations · Advanced glaucoma

\begin{abstract}
Purpose: To evaluate the associations of optical coherence tomography (OCT)-derived macular ganglion cell-inner plexiform layer thickness (mGCIPLT), circumpapillary retinal nerve fiber layer thickness (cpRNFLT), and optic nerve head $(\mathrm{ONH})$ parameters with visual field (VF) sensitivity in advanced glaucoma. Methods: In this cross-sectional study, 102 eyes from 102 patients with advanced glaucoma (defined as a 24-2 VF mean deviation (MD) of $\leq-12 \mathrm{~dB}$ ) were included. mGCIPLT, cpRNFLT, and ONH parameters (including the rim area, average cup-to-disc [C:D] ratio, and vertical $C: D$ ratio) were measured using Cirrus high-definition $O C T$, and 24-2 and 10-2 VF sensitivity tests were performed using standard automated perimetry. Pearson correlations and linear models were used to analyze relationships between OCT-derived parameters and VF parameters. Results: The mGCIPLT and rim area were significantly positively correlated with the 24-2 VF MD, 24-2 VF pattern standard deviation, 24-2 VF visual field index, and 10-2 VF MD, but cpRNFLT was not significantly correlated with VF parameters. In addition, the average and vertical C:D ratios were significantly negatively correlated with VF parameters. The mGCIPLT and rim
\end{abstract}

area were significantly positively correlated with the 10-2 VF $\mathrm{MD}$ ( $r$ ranging between 0.542 and $0.621, p<0.001$ ), while the average and vertical C:D ratios were significantly negatively correlated with the 10-2 VF MD $(r=-0.537, p<0.001$, and $r=-0.428, p<0.001$, respectively). Each $1-\mu \mathrm{m}$ change in the average mGCIPLT was associated with an approximately $0.368-\mathrm{dB}$ change in the 24-2 VF MD and 0.677-dB change in the 10-2 VF MD $\left(R^{2}=0.268, p<0.001\right.$, and $R^{2}=0.385, p<$ 0.001 , respectively). The $10-2$ VF MD showed a significantly stronger association with inferonasal mGCIPLT than did the 24-2 VF MD in advanced glaucoma ( $p=0.007)$. Conclusions: mGCIPLT and ONH parameters were associated with the severity of VF damage and reflected functional damage better than cpRNFLT in advanced glaucoma. Our results suggested that structural measurements of $\mathrm{mGCIPLT}$ and ONH parameters and functional measurement of the 10-2 VF may be useful for monitoring progression in advanced glaucoma.

(c) 2020 S. Karger AG, Basel

\section{Introduction}

Glaucoma has become the most frequent cause of irreversible blindness worldwide and is associated with characteristic structural changes in the optic nerve head $(\mathrm{ONH})$ and retinal nerve fiber layer (RNFL), which are accompanied by functional visual field (VF) loss [1-3]. In karger@karger.com

www.karger.com/ore

(C) 2020 S. Karger AG, Base

Karger'
Xu Zhang

Jiangxi Clinical Research Center for Ophthalmic Disease, Jiangxi Research Institute of Ophthalmology and Visual Science, Affiliated Eye Hospital of Nanchang University 463 Bayi Rd., Nanchang, Jiangxi 330006 (China)

xuzhang19@163.com 
clinical practice and randomized clinical trials, the results of standard automated perimetry (SAP) testing and optical coherence tomography (OCT) imaging have been used as the main outcome measures to evaluate the progression of glaucoma $[4,5]$. OCT is a reliable method to detect glaucoma and has been widely used in the quantitative evaluation of structural parameters, such as circumpapillary retinal nerve fiber layer thickness (cpRNFLT) and macular ganglion cell-inner plexiform layer thickness (mGCIPLT) [6, 7]. Progressive changes in cpRNFLT can be identified objectively by serial analysis of OCT measurements and represent a better structural indicator of VF progression [8]. However, in advanced glaucoma, cpRNFLT has a minimum practical measurement (the so-called floor effect) that restricts its usefulness for detecting disease progression beyond this threshold $[9,10]$. Detection of disease progression is therefore clinically challenging at advanced stages of the disease. Previous studies have demonstrated that the glaucoma discriminating ability of mGCIPLT is comparable to that afforded by cpRNFLT measurement $[7,11,12]$. mGCIPLT is less likely than cpRNFLT to reach the floor measurement in advanced glaucoma, suggesting that mGCIPLT is better for detecting progression $[13,14]$. In a population with advanced glaucoma (defined as an average cpRNFLT of $\leq 60 \mu \mathrm{m}$ ), Lavinsky et al. [15] demonstrated that the rate of change in mGCIPLT was significant at $-0.57 \mu \mathrm{m} /$ year, while that in the rim area was $-0.01 \mathrm{~mm}^{2} /$ year. Shin et al. [16] found that the average rate of change in mGCIPLT was significantly higher in VF progressors than in nonprogressors among patients with moderate-to-advanced glaucoma (defined as a mean deviation $[\mathrm{MD}]$ of $<-6 \mathrm{~dB}$ ), while the average rate of change in cpRNFLT was not significant.

The Humphrey field analyzer (HFA) is one of the most commonly used SAP devices. The 24-2 program tests a total of 54 points that are $6^{\circ}$ apart, and the 10-2 program examines 68 test points that are $2^{\circ}$ apart. The $24-2$ program includes only 12 points located within the central 10 degrees of fixation $[17,18]$ and cannot adequately test the macular region. The rate of MD changes was significantly greater when using the 10-2 VF than when using the 24-2 VF, and evaluation of the 10-2 VF may help provide a better estimation of glaucoma progression in eyes with advanced and severe glaucoma [19].

Evaluating the structural and functional states of the eye is essential to the assessment of glaucoma progression. Multiple studies have used mGCIPLT to show a significant structure-function relationship at the macula in the relatively early stage of glaucoma [20-22]. Structural changes in the macula, such as reductions in MGCIPLT, may be indicators of defects in the central VF (measured using the HFA 30-2 VF test) [23], which have a greater impact than peripheral VF defects on vision-related quality of life (QOL) [24]. One study found a significant association between $\mathrm{mGCIPLT}$ in retinal areas corresponding to the central 4 test points of the HFA 24-2 program and SAP sensitivity in advanced glaucoma [25]. Central vision is often preserved until the very late stages of glaucoma and cannot be accurately assessed unless the VF is monitored using a program specifically designed for detailed measurement of the central $10^{\circ}$, such as the HFA 10-2 VF test [18, 26-28].

However, few studies have evaluated the structurefunction relationship at the macula in advanced glaucoma $[23,29-31]$, and we hypothesized that the mGCIPL and $\mathrm{ONH}$ are better locations than the cpRNFL for monitoring glaucoma progression and that the $10-2 \mathrm{VF}$ test is more suitable than the 24-2 VF test for assessing function in advanced glaucoma. In the present study, we investigated the structure-function relationships between mGCIPLT, cpRNFLT, and ONH parameter measurements obtained using Cirrus high-definition OCT (HDOCT) with the results of 24-2 and 10-2 VF tests performed using SAP in advanced glaucoma patients.

\section{Subjects and Methods}

\section{Subjects}

In this cross-sectional study, all of the advanced glaucoma patients were enrolled after they provided written consent between April 2018 and June 2020 at the Affiliated Eye Hospital of Nanchang University (Jiangxi, China). The authors declare they have no financial or proprietary interests. The study protocol was approved by the Institutional Review Board of our hospital and designed in accordance with the Declaration of Helsinki. All participants received a comprehensive ophthalmological examination, including an annual review of their medical history, best-corrected visual acuity (BCVA) determination, slit-lamp biomicroscopy, intraocular pressure (IOP) measurement using Goldmann applanation tonometry (AT900; Haag-Streit, Köniz, Switzerland), central corneal thickness measurement using Pentacam HR (70900; Oculus, Wetzlar, Germany), gonioscopy (OSMG; Ocular, Bellevue, WA, USA), dilated fundoscopy examination (V90C; Volk, $\mathrm{OH}$, USA), simultaneous stereoscopic optic disc photography (VISUCAM 224; Carl Zeiss Meditec, Jena, Germany), 24-2 VF and 10-2 VF tests with the Humphrey field analyzer (720i; Carl Zeiss Meditec, Dublin, CA, USA), and measurements of mGCIPLT, cpRNFLT, and ONH parameters obtained with HD-OCT (5000; Carl Zeiss Meditec, Dublin, CA, USA).

The inclusion criteria for all subjects were (1) a diagnosis of advanced primary open-angle glaucoma (POAG) or chronic primary angle-closure glaucoma (CPACG) based on a 24-2 VF MD 
of $\leq-12 \mathrm{~dB}$, (2) age $\geq 18$ years, (3) a BCVA of at least 20/50, and (4) a spherical refraction of -6.0 to +3.0 diopters and cylinder correction within \pm 3.0 diopters. POAG was diagnosed in patients with a glaucomatous VF defect in 2 consecutive reliable VF examinations, typical glaucomatous optic neuropathy (i.e., neuroretinal rim thinning, notching, or an RNFL defect), open angles on gonioscopy, and a confirmed untreated IOP $>21 \mathrm{~mm} \mathrm{Hg}[32,33]$. Glaucomatous VF defects were defined as a cluster of 3 or more nonedge contiguous points on a pattern deviation plot at the $p<5 \%$ level (with at least 1 point depressed at the $p<1 \%$ level) confirmed in at least 2 consecutive examinations, a pattern standard deviation (PSD) at the $p<5 \%$ level, or a glaucoma hemifield test result outside normal limits [34]. CPACG was defined as (1) no history of symptoms pertaining to acute angle closure such as ocular or periocular pain, nausea, or intermittent blurry vision with halos; (2) an ocular examination showing no signs of a prior acute attack, for example, a mid-dilated pupil, segmental iris atrophy, and glaukomflecken; (3) chronically elevated IOP (>21 mm Hg); (4) synechial closure of the anterior chamber angle upon gonioscopy; and (5) glaucomatous ONH changes with corresponding VF defects $[35,36]$.

The severity of glaucomatous damage was classified as early, moderate, or advanced according to the Hodapp-Parrish-Anderson criteria [37]. Advanced glaucoma was defined by a baseline 24-2 VF MD of $-12 \mathrm{~dB}$ or less. If both eyes of a patient satisfied the eligibility criteria, then 1 eye was selected randomly for inclusion.

The exclusion criteria were (1) a history of intraocular surgery (except for uncomplicated cataract or glaucoma surgery); coexisting retinal pathology, nonglaucomatous optic neuropathy, uveitis, ocular trauma, or other factors leading to secondary glaucoma; (2) a diagnosis of Parkinson's disease, Alzheimer's disease, or dementia or a history of stroke; (3) diabetic or hypertensive retinopathy; (4) problems other than glaucoma that could affect color vision or a life-threatening disease precluding retention in the study; (5) unreliable VF measurements; and (6) poor-quality OCT scans. Participants with systemic hypertension or diabetes mellitus were included unless they met exclusion criterion number 3.

\section{Visual Field Testing}

VFs were tested using the Swedish interactive thresholding algorithm with 24-2 and 10-2 perimetry after refractive correction with a Goldmann size III target and background luminance (31.5 asb). The 24-2 VF was examined 1st, and the 10-2 VF test was performed later only when the $24-2 \mathrm{VF}$ MD was $-12 \mathrm{~dB}$ or less. The median time between VF examinations was at least 20 min on the same day. Reliable VFs were considered those with $<33 \%$ fixation loss and false-positive and false-negative responses. The MD, PSD, and VF index (VFI) were used for the analysis.

\section{Optical Coherence Tomography Imaging}

All subjects underwent imaging using Cirrus HD-OCT, with 1 optic disc (Optic Disc Cube $200 \times 200$ protocol) scan and 1 macular (Macular Cube $512 \times 128$ protocol) scan acquired by the same operator on the same day. The circumpapillary scan allowed measurement of the cpRNFLT and ONH parameters, whereas the macular scan allowed determination of the mGCIPLT by the ganglion cell analysis (GCA) algorithm. Scans were disqualified if they had a signal strength of $<7$, segmentation errors, or motion artifacts, which were defined as a discontinuity of the blood vessels exceeding the width of 1 major vessel diameter. cpRNFLT (global

Table 1. Demographics and clinical characteristics of the study population

Advanced glaucoma

Age, years

Gender (male, female)

IOP, $\mathrm{mm} \mathrm{Hg}$

CCT, $\mu \mathrm{m}$

Spherical equivalent, diopters

BCVA, logMAR

Type of glaucoma (POAG, CPACG)

VF parameters

24-2 VF MD, dB

24-2 VF PSD, dB

24-2 VF VFI, \%

10-2 VF MD, dB

10-2 VF PSD, dB

cPRNFLT, $\mu m$

Average

Superior

Inferior

Nasal

Temporal

$m G C I P L T, \mu m$

Average

Minimum

Superior

Inferior

Superonasal

Inferonasal

Superotemporal

Inferotemporal

ONH parameters

Rim area, $\mathrm{mm}^{2}$

Average C:D ratio

Vertical C:D ratio

$0.86 \pm 0.06$

$0.85 \pm 0.07$

IOP, intraocular pressure; CCT, central corneal thickness; VF, visual field; $\mathrm{MD}$, mean deviation; $\mathrm{PSD}$, pattern standard deviation; VFI, visual field index; $\mathrm{dB}$, decibels; mGCIPLT, macular ganglion cell-inner plexiform layer thickness; cpRNFLT, circumpapillary retinal nerve fiber layer thickness; ONH, optic nerve head; $\mathrm{C}: \mathrm{D}$ ratio, cup-to-disc ratio; $\mathrm{BCVA}$, best-corrected visual acuity; logMAR, logarithm of the Minimum Angle of Resolution.

and quadrants), mGCIPLT (global and sectorial), and ONH parameters (including the rim area, average C:D ratio, and vertical C:D ratio) were used for the analysis.

\section{Statistical Analyses}

Descriptive statistics included the mean and standard deviation for all continuous variables. The correlations of the mGCIPLT, cpRNFLT, and ONH parameters with VF parameters were investigated using Pearson rank correlation coefficients. The degree of correlation was categorized as strong when $r \geq 0.6$, moderate when 
Table 2. Pearson correlation coefficients of the mGCIPLT, cpRNFLT, and ONH parameters with VF parameters in advanced glaucoma

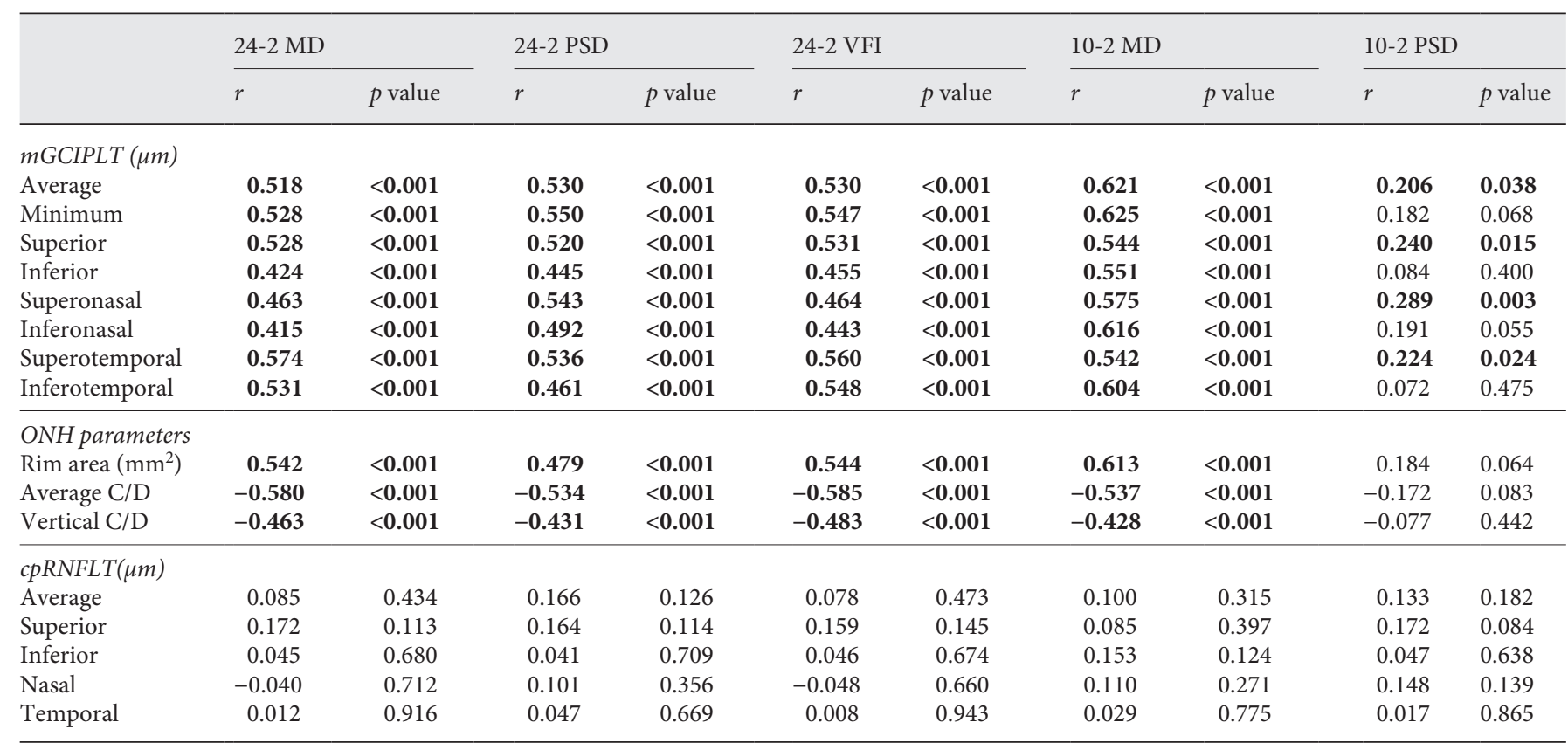

Bold type denotes significance. mGCIPLT, macular ganglion cell-inner plexiform layer thickness; ONH, optic nerve head; cpRNFLT, circumpapillary retinal nerve fiber layer thickness; VF, visual field; MD, mean deviation; PSD, pattern standard deviation; VFI, visual field index.

$0.6>r \geq 0.4$, and mild when $r<0.4$. Linear models were used to investigate univariable associations of the mGCIPLT and $\mathrm{ONH}$ parameters with VF parameters, with the multivariable model fit adjusted for possible confounders (age and the scan quality index). The associations are reported as the coefficient of determination $\left(R^{2}\right)$ of the linear regression models, and the regression coefficient $(\beta)$ was calculated between the mGCIPLT and ONH parameters and VF parameters. The strength of the relationship between structural parameters and the 10-2 VF MD was compared with that of the relationship between structural parameters and the 24-2 VF MD using Steiger's test [38], which is used for comparisons of dependent correlations with a common parameter. A $p$ value of $<0.05$ indicated a statistically significant difference. SPSS V.24.0 (SPSS, Inc., Chicago, IL, USA) and R V.3.6.3 (package "cocor") were used for our statistical analysis.

\section{Results}

\section{Demographics and Clinical Characteristics of the \\ Study Population}

One hundred and twenty-one eyes of 121 participants were evaluated for inclusion in the study. Nineteen subjects with unreliable VF tests or OCT images were excluded; thus, 102 eyes of 102 advanced glaucoma patients were included in this cross-sectional study, 43 of whom had POAG, while 59 patients had CPACG. The demographics and clinical characteristics of the study population are summarized in Table 1. The mean age of the patients was $59.25 \pm 13.58$ years, and the mean baseline IOP was $27.85 \pm$ $11.62 \mathrm{~mm} \mathrm{Hg}$. The mean 24-2 VF MD and 10-2 VF MD were $-26.68 \pm 6.22 \mathrm{~dB}$ and $-22.59 \pm 9.29 \mathrm{~dB}$, and the average cpRNFLT, mGCIPLT, and C:D ratio were $63.48 \pm 9.94$ $\mu \mathrm{m}, 57.26 \pm 8.52 \mu \mathrm{m}$, and $0.86 \pm 0.06 \mu \mathrm{m}$, respectively.

\section{Correlations of the mGCIPLT, cPRNFLT, and}

ONH Parameters with VF Parameters in Advanced

\section{Glaucoma}

The Pearson correlation coefficients of the mGCIPLT, cpRNFLT, and ONH parameters with VF parameters are shown in Table 2. In advanced glaucomatous eyes, the mGCIPLT and ONH parameters were significantly correlated with the 24-2 VF MD, 24-2 VF PSD, 24-2 VF VFI, and 10-2 VF MD $(p<0.001)$. None of the cpRNFLT parameters were significantly correlated with VF parameters. The mGCIPLT and rim area were significantly positively correlated with the 24-2 VF MD ( $r$ ranging between 0.415 and $0.574, p<0.001$ ), while the average and vertical C:D ratios were significantly negatively correlated with the 24-2 VF MD $(r=-0.580, p<0.001$ and $r=-0.463$, $p<0.001$, respectively). Moderate correlations $(|r|$ ranging between 0.431 and $0.550, p<0.001)$ were observed 

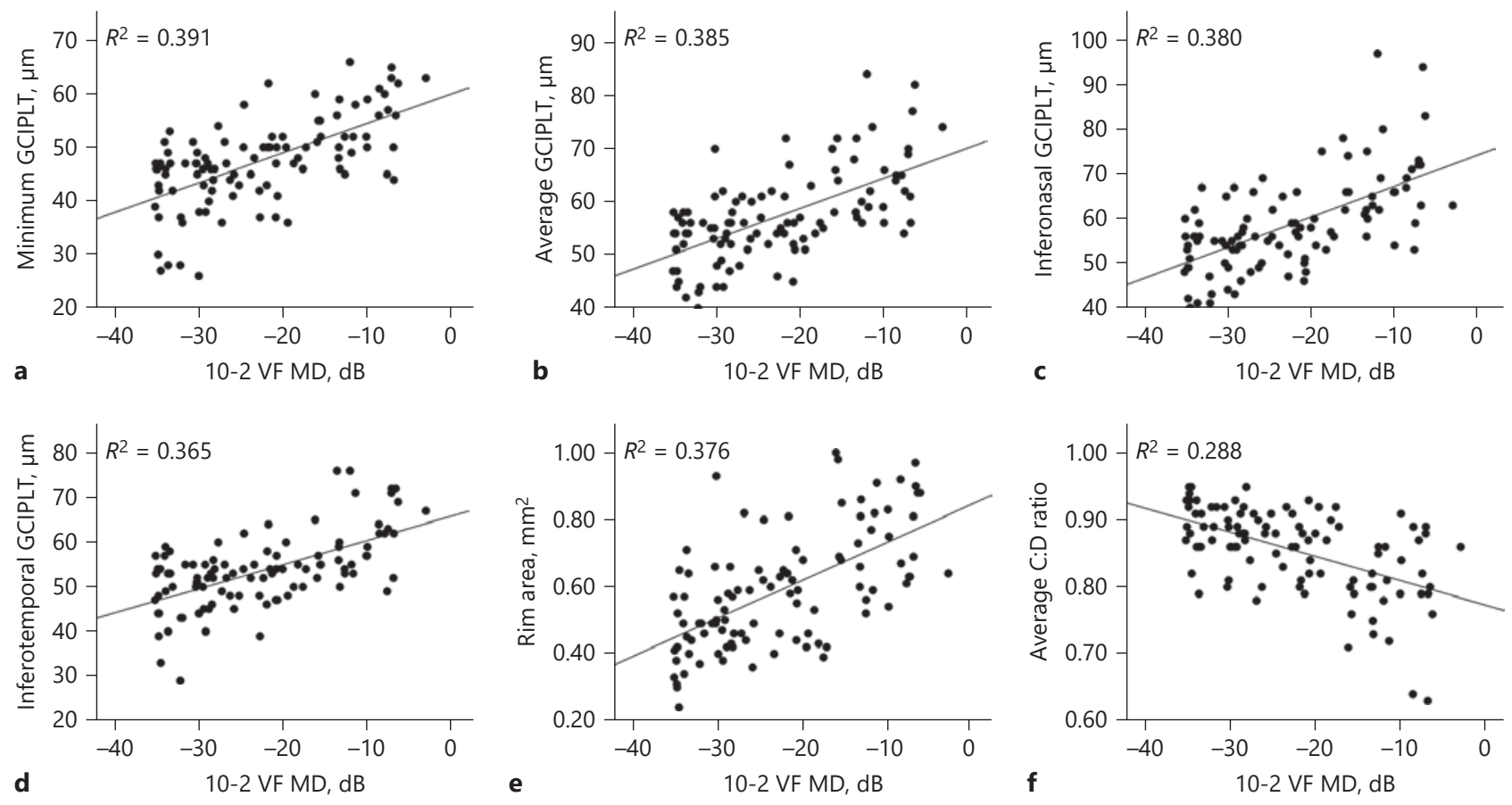

Fig. 1. Scatter plots showing the linear relationships of the mGCIPLT and ONH parameters with the 10-2 VF MD. a The minimum GCIPLT versus the 10-2 VF MD. b The average GCIPLT versus the 10-2 VF MD. c The inferonasal GCIPLT versus the 10-2 VF MD. d The inferotemporal GCIPLT versus the 10-2 VF MD. e The rim area versus the 10-2 VF MD. $f$ The average C:D ratio versus the 10-2 VF MD. mGCIPLT, macular ganglion cellinner plexiform layer thickness; ONH, optic nerve head; VF, visual field; $\mathrm{MD}$, mean deviation; C:D ratio, cupto-disc ratio.

between the mGCIPLT and ONH parameters and the 24-2 VF PSD. The correlations between the mGCIPLT and $\mathrm{ONH}$ parameters and the 24-2 VF VFI were also moderate $(|r|$ ranging between 0.443 and $0.585, p<0.001)$. Moderate or strong correlations were observed between the mGCIPLT and ONH parameters and the 10-2 VF MD. The mGCIPLT and rim area were significantly positively correlated with the 10-2 VF MD ( $r$ ranging between 0.542 and $0.621, p<0.001$ ), while the average and vertical C:D ratios were significantly negatively correlated with the 10-2 VF MD $(r=-0.537, p<0.001$ and $r=$ $-0.428, p<0.001$, respectively). Scatter plots were generated to show the linear relationships between the mGCIPLT and ONH parameters and the 10-2 VF MD (Fig. 1). However, only very mild positive correlations (ranging between 0.206 and $0.289, p<0.05$ ) were observed between the average, superior, superonasal, and superotemporal mGCIPLTs and the 10-2 VF PSD in advanced glaucomatous eyes. Correlations between all cpRNFLT and VF parameters were not significant.
Associations of the mGCIPLT and ONH Parameters with VF Parameters in Advanced Glaucoma:

\section{Univariable Analysis}

Table 3 shows the results of the univariable linear regression models used to evaluate the structure-function relationships between the $\mathrm{mGCIPLT}$ and ONH parameters and the 24-2 VF MD and 10-2 VF MD. The $R^{2}$ values for the associations between mGCIPLT sectors and the 24-2 VF MD ranged from 0.172 (for the inferonasal GCIPL sector) to 0.329 (for the superotemporal GCIPL sector). The $R^{2}$ values for the associations between mGCIPLT sectors and the 10-2 VF MD ranged from 0.294 (for the superotemporal GCIPL sector) to 0.391 (for the minimum GCIPL sector). Each $1-\mu \mathrm{m}$ change in the average mGCIPLT was associated with an approximately $0.368-\mathrm{dB}$ change in the $24-2 \mathrm{VF} \mathrm{MD}$ and $0.677-\mathrm{dB}$ change in the 10-2 VF MD $\left(R^{2}=0.268, p<\right.$ 0.001 and $R^{2}=0.385, p<0.001$, respectively). The $R^{2}$ values for the associations between $\mathrm{ONH}$ parameters and the 24-2 VF MD ranged from 0.215 (for the vertical 
Table 3. Univariable linear regression results describing the relationships of the mGCIPLT and ONH parameters with VF parameters in advanced glaucoma

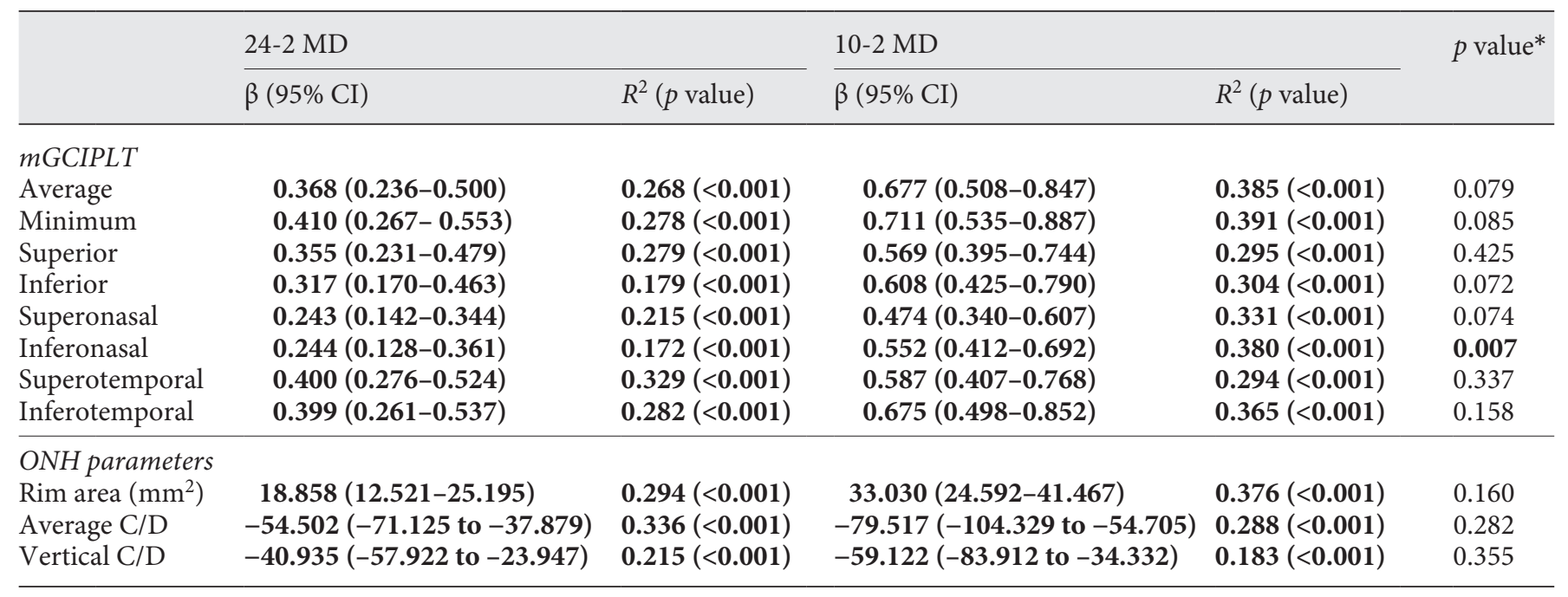

Bold type denotes significance. mGCIPLT, macular ganglion cell-inner plexiform layer thickness; ONH, optic nerve head; VF, visual field; MD, mean deviation. * Comparison of the coefficient of determination $\left(R^{2}\right)$ with structural parameters between 10-2 VF MD and 24-2 VF MD using Steiger's test.

Table 4. Multivariable (adjusted for age and the scan quality index) linear regression results describing the relationships of the mGCIPLT and ONH parameters with VF parameters in advanced glaucoma

\begin{tabular}{|c|c|c|c|c|}
\hline & \multicolumn{2}{|l|}{ 24-2 MD } & \multicolumn{2}{|l|}{ 10-2 MD } \\
\hline & $\beta(95 \% \mathrm{CI})$ & $p$ value & $\beta(95 \% \mathrm{CI})$ & $p$ value \\
\hline \multicolumn{5}{|l|}{$m G C I P L T$} \\
\hline Average & $0.355(0.217-0.492)$ & $<0.001$ & $0.642(0.467-0.817)$ & $<0.001$ \\
\hline Minimum & $0.399(0.250-0.547)$ & $<0.001$ & $0.687(0.507-0.867)$ & $<\mathbf{0 . 0 0 1}$ \\
\hline Superior & $0.340(0.210-0.469)$ & $<0.001$ & $0.522(0.341-0.703)$ & $<0.001$ \\
\hline Inferior & $0.305(0.151-0.459)$ & $<0.001$ & $0.584(0.395-0.773)$ & $<0.001$ \\
\hline Superonasal & $0.228(0.124-0.333)$ & $<0.001$ & $0.438(0.300-0.577)$ & $<\mathbf{0 . 0 0 1}$ \\
\hline Inferonasal & $0.228(0.107-0.349)$ & $<0.001$ & $0.521(0.377-0.665)$ & $<0.001$ \\
\hline Superotemporal & $0.385(0.258-0.513)$ & $<0.001$ & $0.542(0.359-0.724)$ & $<0.001$ \\
\hline Inferotemporal & $0.393(0.254-0.533)$ & $<0.001$ & $0.655(0.479-0.831)$ & $<0.001$ \\
\hline \multicolumn{5}{|l|}{ ONH parameters } \\
\hline Rim area $\left(\mathrm{mm}^{2}\right)$ & $19.160(12.989-25.331)$ & $<0.001$ & $32.797(24.858-40.735)$ & $<0.001$ \\
\hline Average C/D & $-54.140(-70.959$ to -37.321$)$ & $<0.001$ & $-75.666(-100.367$ to -50.965$)$ & $<0.001$ \\
\hline Vertical C/D & $-41.789(-59.348$ to -24.229$)$ & $<0.001$ & $-55.619(-80.696$ to -30.541$)$ & $<0.001$ \\
\hline
\end{tabular}

Bold type denotes significance. mGCIPLT, macular ganglion cell-inner plexiform layer thickness; ONH, optic nerve head; VF, visual field; MD, mean deviation.

C:D ratio) to 0.336 (for the average C:D ratio). The $R^{2}$ values for the associations between mGCIPLT sectors and the 10-2 VF MD ranged from 0.183 (for the vertical C:D ratio) to 0.376 (for the rim area). Each 0.1 change in the average C:D ratio was associated with an approx- imately 5.450- $\mathrm{dB}$ change in the 24-2 VF MD and 7.952$\mathrm{dB}$ change in the 10-2 VF MD $\left(R^{2}=0.336, p<0.001\right.$ and $R^{2}=0.288, p<0.001$, respectively) in advanced glaucoma. The strength of the relationship between structural parameters and the 10-2 VF MD was compared 


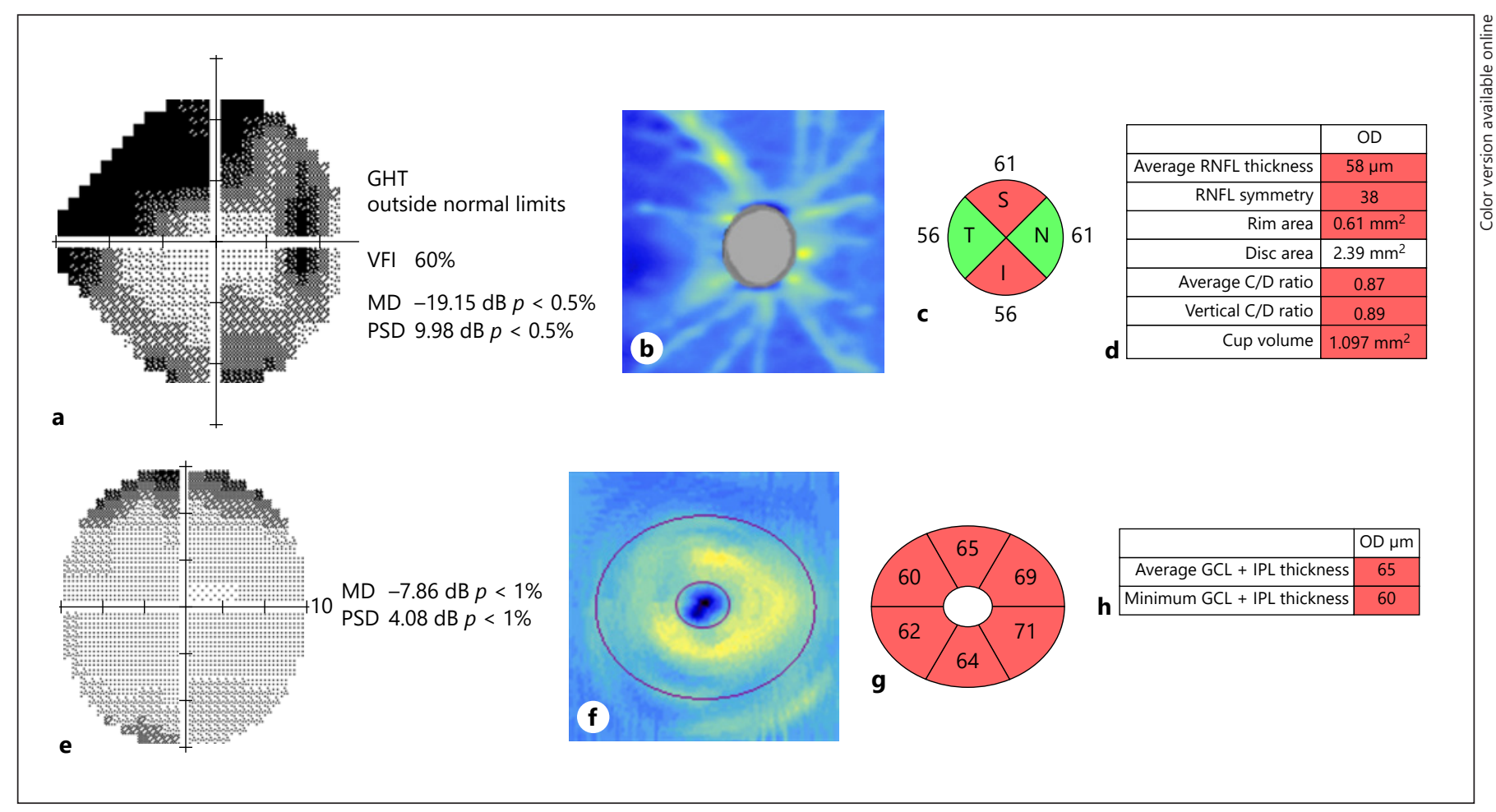

Fig. 2. A representative eye with advanced glaucoma. a The 24-2 VF gray scale printout diagram. b RNFL thickness map. c RNFL thickness in 4 sectors. d ONH parameters. e The 10-2 VF gray scale printout diagram. f GCIPL thickness map. g GCIPL thickness in 6 sectors. $\mathbf{h}$ GCIPL parameters. VF, visual field, GCIPL, ganglion cell-inner plexiform layer; RNFL, retinal nerve fiber layer; ONH, optic nerve head; C/D ratio, cup-to-disc ratio; VFI, visual field index; MD, mean deviation; PSD, pattern standard deviation.

with that of the relationship between structural parameters and the 24-2 VF MD using Steiger's test. Although the associations were stronger between the average, minimum, superior, inferior, superonasal, and inferotemporal mGCIPLTs and the 10-2 VF MD versus the 24-2 VF MD, the differences were not significant. However, the 10-2 VF MD showed a significantly stronger association with inferonasal mGCIPLT than did the 24-2 VF MD in eyes with advanced glaucoma ( $p=$ $0.007)$.

\section{Associations of the $m$ GCIPLT and ONH Parameters with VF Parameters in Advanced Glaucoma: \\ Multivariable Analysis}

The results of multivariable (adjusted for age and the scan quality index) linear regressions between the mGCIPLT and ONH parameters and the 24-2 VF MD and 10-2 VF MD are shown in Table 4. In multivariable analyses, the regression coefficient $(\beta)$ between the mGCIPLT and ONH parameters and the 24-2 VF MD and 10-2 VF MD remained significant after controlling for age and the scan quality index as potential confounding factors. Each $1-\mu \mathrm{m}$ change in the average mGCIPLT was associated with an approximately $0.355-\mathrm{dB}$ change in the 24-2 VF MD $(p<0.001)$ and $0.642-\mathrm{dB}$ change in the $10-2$ VF MD $(p<0.001)$ in advanced glaucoma. Each 0.1 change in the average C:D ratio was associated with an approximately 5.414- $\mathrm{dB}$ change in the 24-2 VF MD $(p<$ $0.001)$ and $7.567-\mathrm{dB}$ change in the 10-2 VF MD $(p<$ 0.001 ) in advanced glaucoma.

\section{An Example of the Findings in This Study}

In 1 representative case of an advanced glaucoma patient, a 34-year-old woman with POAG had a 24-2 VF MD of $-19.15 \mathrm{~dB}$, and the average cpRNFLT, C:D ratio, and $\mathrm{mGCIPLT}$ were $58 \mu \mathrm{m}, 0.87$, and $65 \mu \mathrm{m}$, respectively, as shown in Figure 2. Central VF damage was detected more precisely by the 10-2 test (Fig. 2e) than the 24-2 test (Fig. 2a). The cpRNFLTs (Fig. 2c) at the nasal and temporal sectors fell between the 5th and 95th percentiles of the age-corrected normal distribution, while the mGCIPLTs at all sectors (Fig. $2 \mathrm{~g}$ ) fell below the 1st per- 
centile. The damage at the MGCIPL seemed to be more severe than that observed at the cpRNFL, but more residual thickness was noted at the mGCIPL (Fig. 2f) than at the cpRNFL (Fig. 2b) in the thickness map.

\section{Discussion}

Glaucoma is generally considered a disease in which central visual acuity is relatively well preserved until the late stages of the disease. Detection of disease progression is of paramount importance in the monitoring of patients with advanced glaucoma because they are at a high risk of conditions that threaten visual function. However, monitoring the progression of advanced glaucoma patients is extremely difficult as traditional structural and functional tests have diminished value [14-16, 39].

In the current study, mGCIPLT and ONH parameters were significantly associated with the severity of 10-2 and 24-2 VF loss in advanced glaucoma eyes. In contrast, cpRNFLT showed no significant association. Comparison of the relationships between the mGCIPLT and ONH parameters and VF loss with that between cpRNFLT and VF loss suggests that mGCIPLT and ONH parameters may be clinically useful for evaluating glaucoma severity and may be correlated with functional defects in patients with more advanced glaucoma.

mGCIPLT was more strongly associated with the VF MD than cpRNFLT. Four reasons may account for this finding. First, this finding is likely due to the structure of the macula, especially the papillomacular bundle, which is relatively resistant to glaucomatous damage and is preserved until the advanced stages of the disease [40]. Second, the macula (defined as the central $8^{\circ}$ around the fovea) includes approximately $30 \%$ of all retinal ganglion cells (RGCs) [41] and supplies the inputs to $55-60 \%$ of the primary visual cortex [42]. Unsurprisingly, mGCIPLT is less likely than cpRNFLT to reach the measurement floor in advanced glaucoma because of the high density of RGCs in the macula and their overwhelming representation in the visual cortex. Third, mGCIPLT is measured in the macular area that falls within the 24-2 and 10-2 VFs, whereas the cpRNFL supplies the entire retina, some of which fall outside the VF testing area and may therefore not be as highly correlated with VFs [43]. Finally, VF sensitivity has a significant rate of change and can be detected by the VF test in advanced glaucoma owing to this phenomenon. Kim et al. [23] revealed that all mGCIPLT parameters showed significant associations with macular VF sensitivity (assessed by calculating the average of the

Associations of GCIPL and ONH with VF in Advanced Glaucoma sensitivities at 12 central VF points using the HFA 30-2 VF test) in advanced glaucoma. Shin et al. [44] demonstrated that mGCIPLT was significantly correlated with central VF sensitivity (defined as the average of 12 central points on the HFA 24-2 VF test) in moderate-to-advanced glaucoma. Previous studies $[30,31]$ have also reported that significant correlations were found between mGCIPLT and VF sensitivity loss (measured using the HFA 10-2 VF test) in advanced glaucoma. Our results are consistent with those of the aforementioned studies exploring the structure-function relationship between $\mathrm{mG}$ CIPLT and the central VF in advanced glaucoma.

Changes in $\mathrm{ONH}$ parameters, such as the rim area and C:D ratio (average and vertical), were small but statistically significant in advanced glaucoma [15]. Kostianeva et al. [45] found significant correlations of the rim area and C:D ratio with the HFA 30-2 VF MD in advanced open-angle glaucoma. Lee et al. [46] evaluated correlations between neuroretinal rim area rates of change and VF rates of change in glaucoma patients with a wide range of glaucoma severity and found that a decreased rim area correlated with a worse MD and the mean threshold sensitivities of fast components of VF decay in moderate/ advanced glaucoma. In our study, we found that the rim area and C:D ratio (average and vertical) were significantly associated with VF loss in advanced glaucoma. The changes in the ONH observed in advanced glaucoma may indicate that excavation and neural tissue loss in the optic nerve continue even at this late stage.

The lack of correlation between cpRNFLT and VF sensitivity in advanced glaucoma can be partially attributed to the "floor effect," after which further thinning no longer occurs [10]. The difference in the compositions of the residual mGCIPLT and cpRNFLT might have caused these structures to show different degrees of reserve in demonstrating variation in stages with more severe damage. Another reason for this imperfect relationship is the inability to obtain precise local measurements with which structures are compared with the corresponding functional retinal areas because the axons in the RNFL originate from many different regions of the retina [47]. Our results are consistent with those of earlier studies demonstrating the structure-function relationships between cpRNFLT and VF parameters in advanced glaucoma [23, 31]. In addition, our results indicated that cpRNFLT parameters may not be suitable for evaluating progression in advanced glaucoma patients.

The 10-2 VF MD showed a significantly stronger association with inferonasal mGCIPLT than did the 24-2 VF MD in eyes with advanced glaucoma. Each $1-\mu \mathrm{m}$ 
change in the average mGCIPLT was associated with an approximately $0.368-\mathrm{dB}$ change in the $24-2 \mathrm{VF} \mathrm{MD}$ and $0.677-\mathrm{dB}$ change in the 10-2 VF MD $\left(R^{2}=0.268, p<0.001\right.$ and $R^{2}=0.385, p<0.001$, respectively). First, this result was observed because most of the peripheral points in the VF test are likely to have reached the "floor" in the advanced stage. The inability to detect further changes in these peripheral points may mask the changes that occur in the central points when the MD is estimated by averaging the sensitivity loss across all points based on a $24-2$ VF. Since only a central island of the field remains in these eyes, the changes may be more obvious when the central VF is evaluated with greater resolution (e.g., with the 10-2 strategy) [19]. Tomairek et al. [48] reported that the 10-2 VF test was useful for assessing the residual central visual function in severe cases with absolute central $10^{\circ}$ defects on the 24-2 VF test. Second, the result may be due, at least partly, to an underrepresentation of the macular region when using the 24-2 strategy. The sampling density of the 24-2 VF may be inadequate for estimating visual sensitivity in the macula $[17,26]$.

The structure-function relationships between the mGCIPLT and ONH parameters and the 24-2 VF VFI were significant because the 24-2 VF VFI is less influenced by progressive cataracts [49] and places greater weighting on the centrally located points that correspond to the macular regions most likely to affect visual function [50]. Regression analysis is commonly performed using the VFI parameter to predict the expected VF loss over a given period [51]. Lee et al. [46] evaluated the rates of structural and functional changes among 465 eyes of 338 subjects with different severities of glaucoma and demonstrated that the VFI rates were significantly worse in the more advanced groups $(p<0.001)$. Our results are in agreement with the conclusion of Bambo et al. [52] that the correlations of central vision (the 4 central VF thresholds) and the VFI on the HFA 24-2 VF test with mGCIPLT were moderate and statistically significant in earlyto-severe stages of glaucoma.

In advanced glaucoma eyes, the mGCIPLT and ONH parameters showed moderate significant positive associations with the 24-2 VF PSD. The possible reasons are as follows: the PSD is a global measure used to quantify VF loss and is less affected by media opacities but may improve VF results in eyes with an MD worse than $-20 \mathrm{~dB}$ [19]. The 24-2 VF PSD will therefore be lower or even normal because of the diffuse decrease in VF sensitivity and the difficulty of highlighting local VF defects in advanced glaucoma eyes. However, only very mild positive correlations were observed between the average, superi- or, superonasal, and superotemporal mGCIPLTs and the 10-2 VF PSD in advanced glaucomatous eyes likely because the 10-2 VF PSD fluctuated and became irregular because it sometimes highlighted local VF defects containing denser detection points in the macula. In general, the 24-2 and 10-2 VF PSD are not suitable for evaluating progression in advanced glaucoma patients.

Several studies have evaluated the structure-function relationship between mGCIPLT and VF sensitivity [22, 23,53 , and we added structural parameters such as $\mathrm{ONH}$ parameters, which rendered the structure-function studies more complete and systematic. Some studies have reported a good correlation between $\mathrm{mGCIPLT}$ and central 24-2 VF sensitivity [22, 25, 44], while other studies have reported a good correlation between mGCIPLT and 10-2 VF sensitivity in the relatively early stage of glaucoma [20, $21,54]$. However, we systematically evaluated and compared the relationships between mGCIPLT, cpRNFLT, and $\mathrm{ONH}$ parameters and 24-2 and 10-2 VF parameters in advanced glaucomatous eyes in this study.

Nevertheless, our study has several limitations that should be acknowledged. First, this study had a cross-sectional design. Longitudinal studies are necessary to confirm our findings. Second, potential misclassification of the study groups and inaccurate assessment of disease severity based on the MD of perimetry were possible due to the high test-retest variability of the VF test. Third, although the regression model best fit our data, structural parameters did not completely account for the variation observed in functional loss associated with glaucoma. Fourth, only Asian participants were included in the study and the effect of race on structure-function relationships has not been explored.

At advanced stages of glaucoma, detecting the progression of the disease is often difficult for clinicians because extensive structural damage has occurred. In our study, we found that mGCIPLT and ONH parameters were significantly associated with functional VF status in advanced stages of glaucoma. mGCIPLT may provide more valuable information than cpRNFLT when attempting to understand structure-function relationships in the macular region as the central VF is usually spared until the advanced stages of glaucoma. Evaluation of VF parameters constitutes an important part of progression detection. However, statistical indices (the MD and PSD) can sometimes be misleading in advanced glaucoma due to low reliability and low reproducibility in the 24-2 VF test. In our study, we found that the 10-2 VF MD had a significantly stronger association with inferonasal mGCIPLT than did the 24-2 VF MD in eyes with ad- 
vanced glaucoma and that each $1-\mu \mathrm{m}$ change in the average mGCIPLT was associated with an approximately $0.368-\mathrm{dB}$ change in the 24-2 VF MD and 0.677- $\mathrm{dB}$ change in the 10-2 VF MD $\left(R^{2}=0.268, p<0.001\right.$ and $R^{2}=0.385$, $p<0.001$, respectively), suggesting that the 10-2 VF test may be more suitable than the 24-2 VF test for assessing functional damage in advanced glaucoma.

In conclusion, we found that mGCIPLT and ONH parameters were associated with the severity of VF damage and reflected functional damage better than cpRNFLT in advanced glaucoma. These results suggested that structural measurements of mGCIPLT and $\mathrm{ONH}$ parameters and functional measurement of the 10-2 VF may be useful for monitoring progression in advanced glaucoma.

\section{Statement of Ethics}

This study was approved by the Ethics Committee of the Affiliated Eye Hospital of Nanchang University, Jiangxi, China, and was conducted according to the Declaration of Helsinki. In this cross-sectional study, all of the advanced glaucoma patients were enrolled after they provided written consent between April 2018 and June 2020 at the Affiliated Eye Hospital of Nanchang University (Jiangxi, China).

\section{Conflict of Interest Statement}

The authors report no conflicts of interest.

\section{Funding Sources}

This research project was supported by the National Natural Science Foundation of China (Grant Nos. 81860170, 81271425, and 81260148) and the Jiangxi Provincial Natural Science Foundation (No. 20181ACG70010).

\section{Author Contributions}

H.H. and X.Z. designed the study. H.H., P.L., and X.Y. recruited the participants and collected their data. H.H., X.Y., and W.W. analyzed the data. H.H. wrote the initial draft. W.W. and H.H. revised the manuscript. X.Z. revised the manuscript and obtained funding.

\section{References}

1 Jonas JB, Aung T, Bourne RR, Bron AM, Ritch R, Panda-Jonas S. Glaucoma. Lancet 2017; 390(10108):2183-93.

2 Tham YC, Li X, Wong TY, Quigley HA, Aung T, Cheng CY. Global prevalence of glaucoma and projections of glaucoma burden through 2040: a systematic review and meta-analysis. Ophthalmology. 2014;121(11):2081-90.

3 Bourne RR, Stevens GA, White RA, Smith JL, Flaxman SR, Price H, et al. Causes of vision loss worldwide, 1990-2010: a systematic analysis. Lancet Glob Health. 2013;1(6): e339-49.

4 Hou HW, Lin C, Leung CK. Integrating macular ganglion cell inner plexiform layer and parapapillary retinal nerve fiber layer measurements to detect glaucoma progression. Ophthalmology. 2018;125(6):822-31.

5 Musch DC, Gillespie BW, Lichter PR, Niziol LM, Janz NK. Visual field progression in the Collaborative Initial Glaucoma Treatment Study: the impact of treatment and other baseline factors. Ophthalmology. 2009; 116(2):200-7.

6 Nouri-Mahdavi K, Nowroozizadeh S, Nassiri N, Cirineo N, Knipping S, Giaconi J, et al. Macular ganglion cell/inner plexiform layer measurements by spectral domain optical coherence tomography for detection of early glaucoma and comparison to retinal nerve fi- ber layer measurements. Am J Ophthalmol. 2013;156(6):1297-1307.e2.

7 Mwanza JC, Durbin MK, Budenz DL, Sayyad FE, Chang RT, Neelakantan A, et al. Glaucoma diagnostic accuracy of ganglion cell-inner plexiform layer thickness: comparison with nerve fiber layer and optic nerve head. Ophthalmology. 2012;119(6):1151-8.

8 Yu M, Lin C, Weinreb RN, Lai G, Chiu V, Leung CK. Risk of visual field progression in glaucoma patients with progressive retinal nerve fiber layer thinning: a 5-year prospective study. Ophthalmology. 2016;123(6):1201-10.

9 de Moraes CG, Liebmann JM, Medeiros FA, Weinreb RN. Management of advanced glaucoma: characterization and monitoring. Surv Ophthalmol. 2016;61(5):597-615.

10 Hood DC, Kardon RH. A framework for comparing structural and functional measures of glaucomatous damage. Prog Retin Eye Res. 2007;26(6):688-710.

11 Mwanza JC, Budenz DL, Godfrey DG, Neelakantan A, Sayyad FE, Chang RT, et al. Diagnostic performance of optical coherence tomography ganglion cell: inner plexiform layer thickness measurements in early glaucoma. Ophthalmology. 2014;121(4):849-54.

12 Takayama K, Hangai M, Durbin M, Nakano $\mathrm{N}$, Morooka S, Akagi T, et al. A novel method to detect local ganglion cell loss in early glau- coma using spectral-domain optical coherence tomography. Invest Ophthalmol Vis Sci. 2012;53(11):6904-13.

13 Bowd C, Zangwill LM, Weinreb RN, Medeiros FA, Belghith A. Estimating optical coherence tomography structural measurement floors to improve detection of progression in advanced glaucoma. Am J Ophthalmol. 2017; 175:37-44.

14 Belghith A, Medeiros FA, Bowd C, Liebmann JM, Girkin CA, Weinreb RN, et al. Structural change can be detected in advanced-glaucoma eyes. Invest Ophthalmol Vis Sci. 2016; 57(9):ОCT511-8.

15 Lavinsky F, Wu M, Schuman JS, Lucy KA, Liu $\mathrm{M}$, Song $\mathrm{Y}$, et al. Can macula and optic nerve head parameters detect glaucoma progression in eyes with advanced circumpapillary retinal nerve fiber layer damage? Ophthalmology. 2018;125(12):1907-12.

16 Shin JW, Sung KR, Lee GC, Durbin MK, Cheng D. Ganglion cell-inner plexiform layer change detected by optical coherence tomography indicates progression in advanced glaucoma. Ophthalmology. 2017;124(10):1466-74.

17 Park SC, Kung Y, Su D, Simonson JL, Furlanetto RL, Liebmann JM, et al. Parafoveal scotoma progression in glaucoma: Humphrey 10-2 versus $24-2$ visual field analysis. Ophthalmology. 2013;120(8):1546-50. 
18 Park HY, Hwang BE, Shin HY, Park CK. Clinical clues to predict the presence of parafoveal scotoma on Humphrey 10-2 visual field using a Humphrey 24-2 visual field. Am J Ophthalmol. 2016;161:150-9.

19 Rao HL, Begum VU, Khadka D, Mandal AK, Senthil S, Garudadri CS. Comparing glaucoma progression on 24-2 and 10-2 visual field examinations. PLoS One. 2015; 10(5): e0127233.

20 Rao HL, Qasim M, Hussain RS, Januwada M, Pillutla LN, Begum VU, et al. Structure-function relationship in glaucoma using ganglion cell-inner plexiform layer thickness measurements. Invest Ophthalmol Vis Sci. 2015; 56(6):3883-8

21 Rao HL, Januwada M, Hussain RS, Pillutla LN, Begum VU, Chaitanya A, et al. Comparing the structure-function relationship at the macula with standard automated perimetry and microperimetry. Invest Ophthalmol Vis Sci. 2015;56(13):8063-8.

22 Shin HY, Park HY, Jung KI, Park CK. Comparative study of macular ganglion cell-inner plexiform layer and peripapillary retinal nerve fiber layer measurement: structurefunction analysis. Invest Ophthalmol Vis Sci. 2013;54(12):7344-53.

23 Kim KE, Park KH, Jeoung JW, Kim SH, Kim DM. Severity-dependent association between ganglion cell inner plexiform layer thickness and macular mean sensitivity in open-angle glaucoma. Acta Ophthalmol. 2014;92(8): e650-6.

24 Medeiros FA, Gracitelli CP, Boer ER, Weinreb RN, Zangwill LM, Rosen PN. Longitudinal changes in quality of life and rates of progressive visual field loss in glaucoma patients. Ophthalmology. 2015;122(2):293-301.

25 Araie M, Murata H, Iwase A, Hangai M, Sugiyama K, Yoshimura N. Differences in relationship between macular inner retinal layer thickness and retinal sensitivity in eyes with early and progressed glaucoma. Invest Ophthalmol Vis Sci. 2016;57(4):1588-94.

26 Traynis I, De Moraes CG, Raza AS, Liebmann JM, Ritch R, Hood DC. Prevalence and nature of early glaucomatous defects in the central $10^{\circ}$ of the visual field. JAMA Ophthalmol. 2014;132(3):291-7.

27 De Moraes CG, Hood DC, Thenappan A, Girkin CA, Medeiros FA, Weinreb RN, et al. 24-2 visual fields miss central defects shown on 10-2 tests in glaucoma suspects, ocular hypertensives, and early glaucoma. Ophthalmology. 2017;124(10):1449-56.

28 Grillo LM, Wang DL, Ramachandran R, Ehrlich AC, De Moraes CG, Ritch R, et al. The 24-2 visual field test misses central macular damage confirmed by the $10-2$ visual field test and optical coherence tomography. Transl Vis Sci Technol. 2016;5(2):15.
29 Mohammadzadeh V, Rabiolo A, Fu Q, Mo rales E, Coleman AL, Law SK, et al. Longitudinal macular structure-function relationships in glaucoma. Ophthalmology. 2020; 127(7):888-900.

30 Lee JW, Morales E, Sharifipour F, Amini N, $\mathrm{Yu}$ F, Afifi AA, et al. The relationship between central visual field sensitivity and macular ganglion cell/inner plexiform layer thickness in glaucoma. Br J Ophthalmol. 2017;101(8): 1052-8.

31 Sung MS, Heo H, Park SW. Structure-function relationship in advanced glaucoma after reaching the RNFL floor. J Glaucoma. 2019; 28(11):1006-11.

32 Tang B, Li S, Han J, Cao W, Sun X. Associations between blood cell profiles and primary open-angle glaucoma: a retrospective casecontrol study. Ophthalmic Res. 2020;63(4): 413-22.

33 Hao L, Xiao H, Gao X, Xu X, Liu X. Measurement of structural parameters of the lamina cribrosa in primary open-angle glaucoma and chronic primary angle-closure glaucoma by optical coherence tomography and its correlations with ocular parameters. Ophthalmic Res. 2019;62(1):36-45.

34 Brusini P, Johnson CA. Staging functional damage in glaucoma: review of different classification methods. Surv Ophthalmol. 2007; 52(2):156-79.

35 Chen HJ, Wang X, Yan YJ, Wu LL. Postiridotomy ultrasound biomicroscopy features in the fellow eye of Chinese patients with acute primary angle-closure and chronic primary angle-closure glaucoma. J Glaucoma. 2015; 24(3):233-7.

36 Foster PJ, Buhrmann R, Quigley HA, Johnson GJ. The definition and classification of glaucoma in prevalence surveys. Br J Ophthalmol. 2002;86(2):238-42.

37 Hodapp E, Parrish RK II, Anderson DR. Clinical decisions in glaucoma. St. Louis: Mosby; 1993. p. 52-61.

38 Steiger JH. Tests for comparing elements of a correlation matrix. Psychol Bull. 1980;87(2): 245-51.

39 Gardiner SK, Swanson WH, Goren D, Mansberger SL, Demirel S. Assessment of the reliability of standard automated perimetry in regions of glaucomatous damage. Ophthalmology. 2014;121(7):1359-69.

40 Hood DC, Raza AS, de Moraes CG, Liebmann JM, Ritch R. Glaucomatous damage of the macula. Prog Retin Eye Res. 2013;32:1-21.

41 Curcio CA, Allen KA. Topography of ganglion cells in human retina. J Comp Neurol. 1990;300(1):5-25.

42 McFadzean R, Brosnahan D, Hadley D, Mutlukan E. Representation of the visual field in the occipital striate cortex. Br J Ophthalmol. 1994;78(3):185-90.
43 Zhang X, Dastiridou A, Francis BA, Tan O, Varma R, Greenfield DS, et al. Baseline Fourier-Domain optical coherence tomography structural risk factors for visual field progression in the advanced imaging for glaucoma study. Am J Ophthalmol. 2016;172:94-103.

44 Shin JW, Lee J, Kwon J, Jo Y, Jeong D, Shon $\mathrm{G}$, et al. Relationship between macular vessel density and central visual field sensitivity at different glaucoma stages. Br J Ophthalmol. 2019;103(12):1827-33

45 Kostianeva SS, Konareva-Kostianeva MI, Atanassov MA. Relationship between visual field changes and optical coherence tomography measurements in advanced open-angle glaucoma. Folia Med. 2016;58(3):174-81.

46 Lee JW, Kim EA, Otarola F, Morales E, Yu F, Afifi AA, et al. The fast component of visual field decay rate correlates with disc rim area change throughout the entire range of glaucomatous damage. Invest Ophthalmol Vis Sci. 2015;56(10):5997-6006.

47 Raza AS, Cho J, de Moraes CG, Wang M, Zhang X, Kardon RH, et al. Retinal ganglion cell layer thickness and local visual field sensitivity in glaucoma. Arch Ophthalmol. 2011; 129(12):1529-36

48 Tomairek RH, Aboud SA, Hassan M, Mohamed AH. Studying the role of 10-2 visual field test in different stages of glaucoma. Eur J Ophthalmol. 2019;30(4):706-13.

49 Rao HL, Jonnadula GB, Addepalli UK, Senthil S, Garudadri CS. Effect of cataract extraction on Visual Field Index in glaucoma. J Glaucoma. 2013;22(2):164-8.

50 Bengtsson B, Heijl A. A visual field index for calculation of glaucoma rate of progression. Am J Ophthalmol. 2008;145(2):343-53.

51 Bengtsson B, Patella VM, Heijl A. Prediction of glaucomatous visual field loss by extrapolation of linear trends. Arch Ophthalmol. 2009; 127(12):1610-5.

52 Bambo MP, Güerri N, Ferrandez B, Cameo B, Fuertes I, Polo V, et al. Evaluation of the macular ganglion cell-inner plexiform layer and the circumpapillary retinal nerve fiber layer in early to severe stages of glaucoma: correlation with central visual function and visual field indexes. Ophthalmic Res. 2017;57(4):216-23.

53 Mwanza JC, Oakley JD, Budenz DL, Chang RT, Knight OJ, Feuer WJ. Macular ganglion cell-inner plexiform layer: automated detection and thickness reproducibility with spectral domain-optical coherence tomography in glaucoma. Invest Ophthalmol Vis Sci. 2011; 52(11):8323-9.

54 Ohkubo S, Higashide T, Udagawa S, Sugiyama K, Hangai M, Yoshimura N, et al. Focal relationship between structure and function within the central 10 degrees in glaucoma. Invest Ophthalmol Vis Sci. 2014;55(8):5269-77. 